\title{
EL USO DE LOS SISTEMAS DE RESPUESTA INTERACTIVA COMO HERRAMIENTA PARA FAVORECER EL APRENDIZAJE PROACTIVO EN INGENIERÍA
}

\section{The Use of Interactive Response Systems as a Tool to Favor Proactive Learning IN ENGINEERING}

\author{
Alberto Jesús Perea Moreno ${ }^{1}$, María Jesús Aguilera Ureña ${ }^{1}$, \\ Ana María Laguna Luna ${ }^{1}$, José Luis de la Cruz Fernández ${ }^{1}$, \\ Manuel Torres Roldán ${ }^{1}$, Jesús Torres Castro ${ }^{1}$, \\ María Carmen Sol Prieto ${ }^{1}$, Gema Guzmán Díaz ${ }^{1}$, \\ Carmen de la Cruz Lovera ${ }^{1}$, José Miguel Martínez Valle',

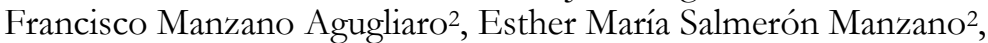 \\ Francisco Gil Montoya², Alfredo Alcayde García ${ }^{2}$ \\ aperea@uco.es \\ ${ }^{1}$ Universidad de Córdoba \\ 2 Universidad de Almería
}

Received: 22/06/2017 Accepted: 20/02/2018

\section{Resumen}

El desarrollo de las tecnologías de la información y la comunicación (TIC) ha permitido el surgimiento de herramientas didácticas en el campo de la educación, proporcionando herramientas prácticas para apoyar a las clases presenciales. En este contexto, los sistemas electrónicos de respuesta estudiantil pueden ser útiles para introducir un elemento tecnológico motivador en las lecciones, así como una nueva metodología.

En este estudio, además del uso de los sistemas de respuesta interactiva o clickers, se ha introducido la tecnología de aprendizaje móvil mediante la elaboración de una metodología de uso de las herramientas Kahoot y Telegram en la asignatura "Fundamentos Físicos en la Ingeniería II" del Grado en Ingeniería Electrónica Industrial. El Departamento de Física Aplicada de la Universidad de Córdoba tiene una amplia experiencia en el uso de Clickers en clases teóricas con grupos grandes para diferentes grados universitarios, pero ahora el uso de tecnologías móviles de aprendizaje se ha introducido en grupos de tamaño medio para clases prácticas. Usando esta nueva metodología, los estudiantes de grupos medianos realizan un cuestionario durante la lección de resolución de problemas, donde utilizaron sus conocimientos adquiridos durante la clase. La realización del cuestionario permite a los profesores evaluar en tiempo real el nivel del estudiante y utilizar la retroalimentación para abordar los problemas iniciales y los malentendidos. Los resultados muestran que los sistemas de respuesta interactiva son altamente valorados por los estudiantes, que lo perciben como una herramienta para mejorar el aprendizaje y aumentar la competencia en el aula.

Palabras clave: Aprendizaje mediante dispositivos móviles; Aprendizaje proactivo; Sistema interactivo de respuesta; Educación superior; Innovación.

\section{Abstract}

The development of information and communication technologies (ICT) has enabled the emergence of teaching tools in the education field, providing practical tools to support face-to-face classes. In this context, electronic student response systems can be useful for introducing a motivating technological element into the lessons, as well as a new methodology.

In this study, in addition to the use of interactive response systems or clickers, mobile learning technology has been introduced by developing a methodology for using the Kahoot and Telegram tools in the subject "Physical Foundations of Engineering II" of Electronic Engineering Degree. Department of Applied Physics of University of Cordoba has a broad experience using Clickers in theory classes with large groups for different university degrees, but now the use of mobile learning technologies has been introduced in medium-sized groups for practical classes. Using this new methodology, students of medium-sized groups perform a quiz during the problem solving lesson, where they utilized their knowledge gained during the class. The completion of the quiz allows teachers to assess the student's level in real time, and to use the feedback to address initial problems and misunderstandings.

Results show the interactive response systems are highly valued by students, who perceive it as a tool to improve learning and increase competition in the classroom.

Keywords: Mobile Learning technology; Proactive learning; Interactive response system; Higher education; innovation.

\section{INTRODUCCIÓN}

Hoy en día, la investigación sobre metodologías de enseñanza muestra los beneficios de los enfoques de aprendizaje activo (LUCKE, T., 2017). La evolución y el desarrollo de las TIC han permitido el desarrollo de nuevas herramientas didácticas de educación, ofreciendo soluciones para acciones de formación a distancia, pero también Clases de apoyo [WAHEED, M., 2016]. En este contexto, los sistemas electrónicos de respuesta personal de los estudiantes son presentados como un elemento tecnológico motivador, siendo muy útil en el aula [KULIKOVSKIKH, I.M., 2017$].$

Las investigaciones realizadas sobre el uso de sistemas remotos que facilitan la respuesta anónima del alumno en el aula, demuestran las ventajas relativas al aprendizaje e involucración de los alumnos [SHAPIRO, A.M., 2017]. Así, los sistemas de respuesta interactiva son una tecnología utilizada para promover el aprendizaje activo [KATZ, L., 2017]. 
El Departamento de Física Aplicada de la Universidad de Córdoba, como resultado de Proyectos de Innovación de convocatorias previas, tiene experiencia en la utilización de mandos de respuesta interactiva Turning Point en clases de Fundamentos Físicos en primer curso de Ingeniería. En concreto, este proyecto se plantea a partir de los resultados obtenidos en el proyecto "Utilización de un sistema de respuesta interactiva en la prácticas de laboratorio de Fundamentos Físicos de la Ingeniería (código 2015-2-5015)" de la convocatoria 2015-2016 de Proyectos de Innovación, en el que se ha llevado a cabo una experiencia de uso de los mandos en prácticas de laboratorio, fomentando el aprendizaje proactivo del alumno. De este modo, la herramienta permite la evaluación del aprendizaje previo del alumno, pero también supone un elemento de retroalimentación para el profesor, ya que permite identificar en el momento si el alumno ha asimilado los conceptos al conocer las respuestas que se han realizado a cada pregunta.

Así, a partir de los buenos resultados obtenidos, como primer objetivo del presente proyecto se ha pretendido extender la metodología en las prácticas de laboratorio de diferentes asignaturas que se imparten en el Departamento de Física Aplicada.

Sin embargo, para la consecución del objetivo planteado existe el inconveniente de que el sistema de mandos Turning Point disponible es insuficiente para ser utilizado en varias asignaturas a la vez, con elevado número de grupos de prácticas por cada una de ellas, coincidiendo a veces horarios de dos grupos distintos, en diferentes laboratorios.

Por otro lado, últimamente se está extendiendo el uso de la tecnología Mobile Learning o m-learning, tecnología que se puede definir como la modalidad educativa que facilita la construcción del conocimiento, la resolución de problemas de aprendizaje y el desarrollo de destrezas o habilidades diversas de forma autónoma y ubicua gracias a la mediación de dispositivos móviles portables [BRIZ-PONCE, L., 2017]. El dispositivo móvil está plenamente integrado en la vida cotidiana de los jóvenes, por lo que se puede plantear su uso para fines académicos. En el contexto del presente proyecto, se han encontrado diversas plataformas para estos dispositivos como Poll Everywhere, Socrative, Kahoot, Mentimeter, Quiz, Socket o Verso, que permiten crear encuestas en tiempo real que se pueden responder de diferentes modos a través del teléfono móvil. Así, estas plataformas integradas en el teléfono móvil del alumno se pueden plantear como una alternativa o complemento al sistema de respuesta interactiva Turning Point. Así, en el presente estudio se plantea como segundo objetivo analizar la potencialidad del teléfono móvil como dispositivo de respuesta en el aula.

\section{OBJETIVOS}

De acuerdo con lo expuesto en el punto anterior, los objetivos del presente proyecto son:

- Análisis de la potencialidad del teléfono móvil como dispositivo de respuesta en el aula, como alternativa o complemento al sistema de respuesta interativa/clickers usando el software Turning Point.

- Implementación de la metodología docente basada en el uso de los sistemas de respuesta interactiva en las prácticas de laboratorio de diferentes asignaturas impartidas en el Departamento de Física Aplicada de la Universidad de Córdoba, y valoración de la misma.

\section{Metodología}

Con el proyecto se ha implementado una nueva metodología de trabajo en laboratorio, fomentando el aprendizaje proactivo del alumno. La disponibilidad de laboratorios es muy limitada, por haber muchos grupos y muchas titulaciones con la asignatura de Fundamentos Físicos en la Ingeniería en el primer curso. Por ello, el tiempo disponible para cada alumno en el laboratorio es muy reducido, y se debe aprovechar al máximo. Con la nueva metodología se se fomenta la actitud activa del alumno en el laboratorio, empezando por trabajar el guion de la práctica con antelación a cada sesión práctica. Para fomentar y controlar este trabajo individual se han realizado cuestionarios mediante el uso de los mandos interactivos de respuesta al inicio de la sesión práctica, donde el alumno deba reflejar los conocimientos mínimos necesarios para realizar la práctica en condiciones adecuadas. Estos cuestionarios previos han servido como autoevaluación para alumno, evaluación del trabajo previo para el profesor, planteándose como herramienta que ha permitido la retroalimentación, con la que el profesor detectaba debilidades de los alumnos, y los alumnos podían resolver errores previos. De esta manera, el alumno ha afrontado la práctica con mayor posibilidad de aprovechamiento de la misma.

Por otro lado, la metodología incluye también la realización de cuestionarios al final de la sesión práctica, con los que el profesor pueda realizar la evaluación sobre el nivel de aprovechamiento del trabajo del alumno en el laboratorio, disponiendo así de una herramienta de evaluación más objetiva sobre las prácticas de laboratorio. En este proyecto los mandos no se han utilizado de manera anónima como en experiencias previas, sino asignando un mando concreto a cada alumno, con lo que se ha obtenido información de cada alumno permitiendo la evaluación continua de las prácticas, y conocer su evolución.

En el desarrollo del presente estudio se han distinguido las siguientes fases:

Primera fase. Revisión de plataformas disponibles para utilización del teléfono móvil como dispositivo de respuesta interactivo. Elección de la/s platafoma/s más adecuada/s al objeto del proyecto.

Segunda fase. Desarrollo de una estrategia para la implementación de la metodología de utilización de dispositivos de respuesta interactiva en asignaturas prácticas del Departamento de Física Aplicada.

Tercera fase. Diseño de material docente. 
Cuarta fase. Diseño de metodología para la validación de la herramienta. Se pasaron encuestas para valorar la opinión de alumnado y profesorado sobre el uso de ambas metodologías.

Quinta fase. Implementación de ambas metodologías en las diferentes sesiones prácticas que se hayan decidido en la Primera Etapa. Obtención de datos sobre valoración de la utilidad de las metodologías en la última sesión de cada grupo.

Sexta fase. Obtención de conclusiones.

\section{DESCRIPCIÓN}

Una vez realizada por el equipo de trabajo la revisión de plataformas disponibles para utilización del teléfono móvil como dispositivo de respuesta interactivo, se eligieron como mejores adaptabas a los objetivos definidos en el proyecto:

\subsection{KAHOOT}

Kahoot es una herramienta para utilizar la tecnología para administrar cuestionarios, discusiones o encuestas. Es un sistema de respuesta basado en el juego de aula jugado por toda la clase en tiempo real. Las preguntas de opción múltiple se proyectan en la pantalla. Kahoot es un sistema de respuesta que crea un compromiso de aprendizaje motivando al alumnado a través de un juego de aula. Los alumnos contestan las preguntas con su smartphone, tablet u ordenador con el objetivo de crear un ambiente educativo motivador y con el objetivo de asimilar conocimientos de forma divertida.

A través de la plataforma web Kahoot, el profesor puede crear cuestionarios, discusiones o encuestas que pueden contar con imágenes o vídeos aclaratorios que complementen el contenido académico (Figura 1).

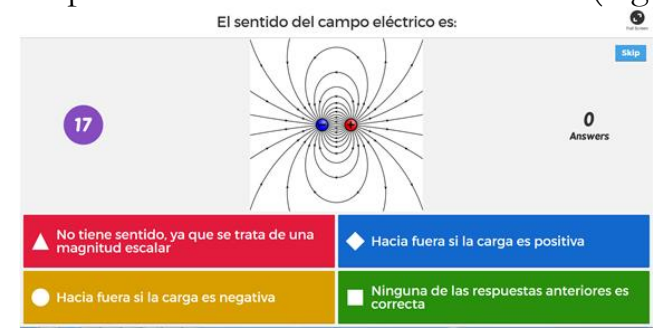

1. Ejemplo de cuestión con cuatro posibles respuestas.

Una vez que el cuestionario ha sido creado, el profesor lo proyecta en el aula y los alumnos acceden a la plataforma desde un ordenador, móvil o tablet, desde el cual emiten la respuesta en tiempo real. Una vez transcurrido el tiempo límite para que los alumnos puedan responder a la pregunta, se emite una tabla de resultados y posiciones con las puntuaciones obtenidas por los alumnos en función de si han acertado la respuesta a la cuestión y el tiempo que han tardado en emitir dicha respuesta. Mientras el docente explica conceptos y repasa contenidos explicados en el tema correspondiente.

Como experiencia, en la asignatura Fundamentos Físicos en la Ingeniería II del Grado en Ingeniería Electrónica de la Universidad de Córdoba, se puso en práctica este sistema con objeto de repasar los contenidos explicados por el profesor (Figura 2).

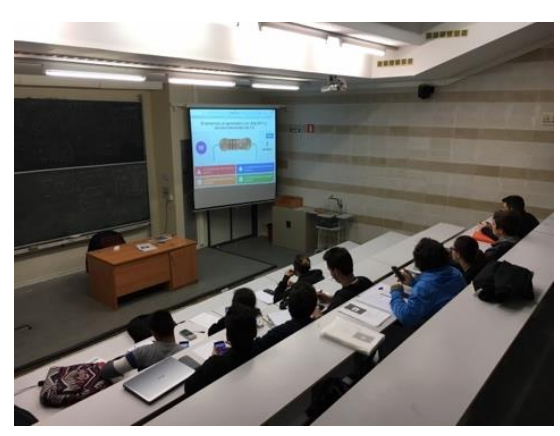

2. Estudiantes usando Kahoot durante las clases prácticas.

\subsection{TELEGRAM}

Se ha evaluado una aplicación gratuita para el aprendizaje en modo M-learning en la educación superior creada por profesores de la Universidad de Almería e integrantes de este proyecto. Un bot Telegram llamado Quiz se ha utilizado en las clases prácticas de Fundamentos Físicos en la Ingeniería I del Grado en Ingeniería Electrónica de la Universidad de Córdoba. El objetivo es facilitar el aprendizaje proactivo de los estudiantes en la educación superior. ZquizUALbot es un bot desarrollado para Telegram (la aplicación de mensajería instantánea), que permite al profesor realizar o programar, muy fácilmente, el cuestionario que se entregará en tiempo real en todos los teléfonos inteligentes del grupo. En esta primera versión, el profesor, sólo tiene que escribir al bot un tipo de comandos de consulta o acción para interactuar con el bot. Una vez seleccionado el 
tipo de cuestionario, el bot prepara una vista previa para el profesor, que, si se acepta, indica un código de activación que el profesor debe proporcionar a los alumnos asistentes verbalmente o escrito en una pizarra. Por lo tanto, una vez que el código fue validado, el estudiante puede enviar la respuesta y se le permite escribir una nota asociada con el cuestionario con un texto libre. Como retroalimentación el profesor recibe respuestas en tiempo real y por lo tanto puede consultar el progreso de la explicación al tiempo que motiva la atención y por lo tanto la participación del grupo de alumnos presentes en la clase.

El software desarrollado es gratuito y flexible de implementar ya que los estudiantes universitarios suelen tener teléfono inteligente.

\subsection{Clickers (TURNING POINT)}

Además, de las plataformas basadas en la utilización del teléfono móvil como dispositivo de respuesta interactivo, se repitieron experiencias mediante el uso de clickers y el software Turnin Point en la asignatura Fundamentos Físicos de la Ingeniería del Grado en Ingeniería Forestal.

Al comienzo de la sesión práctica, el profesor planteaba un cuestionario inicial, usando el software Turning Point. En la Figura 3 se ilustra una sesión práctica con el sistema expuesto. Los alumnos disponen de un tiempo límite para emitir su respuesta con los clickers a la pregunta presenta en cada diapositiva. Alcanzado el tiempo límite, se muestra en la pantalla, la respuesta correcta y el porcentaje de alumnos que han acertado o han elegido otra respuesta no correcta.

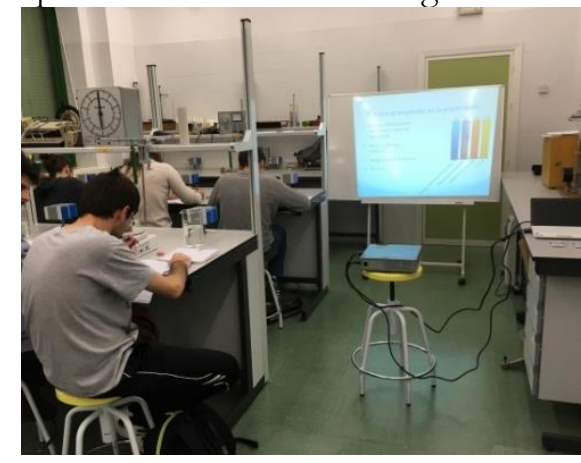

3. Ejemplo de sesión práctica en laboratorio usando clickers.

Finalizadas las prácticas de laboratorio, el profesor plantea a los alumnos otro cuestionario para comprar el nivel de asimilación aprovechamiento durante la sesión de prácticas. El sistema almacena cada respuesta realizada por cada alumno en una base de datos, de forma que la calificación obtenida puede ser utilizada posteriormente en la evaluación de la asignatura. Así mismo, el profesor puede dar explicaciones complementarias en base a los resultados que se obtienen de las respuestas emitidas en cada pregunta por los alumnos.

\section{RESULTADOS OBTENIDOS}

La metodología presentada debe ser evaluada para poder ser validada, por ello se recurrió a valorar los siguientes aspectos:

$\checkmark$ Encuesta de satisfacción del alumnado. Esta encuesta se realizó mediante un link a un formulario de google (google forms).

$\checkmark$ Asistencia a clase: Aumento o disminución.

Para conocer la opinión de los alumnos sobre esta experiencia, se les ha sometido a una encuesta de opinión anónima. En dicha encuesta se pide a los alumnos que muestren su grado de acuerdo/desacuerdo con diferentes afirmaciones sobre el uso del sistema de respuesta interactiva en las prácticas de laboratorio, utilizando una escala Likert 1 (total desacuerdo)-5 (acuerdo total). Se les plantearon las siguientes preguntas:

1. Los sistemas y plataformas de respuesta interactiva son un recurso docente motivador.

2. El uso de los sistemas y plataformas de respuesta interactiva hace que adopte una actitud más activa en el aula.

3. El uso de los sistemas y plataformas de respuesta interactiva favorece una retroalimentación inmediata profesor-alumno.

4. El uso de los mandos interactivos fomenta el trabajo en grupo.

5. Valoración global del recurso educativo.

De los resultados obtenidos de la encuesta de opinión anónima sobre las nuevas metodologías docentes (Figura 4), se observa que el $81.75 \%$ de los alumnos se muestran de acuerdo o muy de acuerdo con esta metodología, suponiendo un recurso motivador, con una valoración media ponderada de 4.09 sobre 5. El 80\% de los alumnos encuestados piensan que el uso de estas tecnologías les involucra con una actitud más activa en el aula. Por otra lado, el 82.75\% de los alumnos piensan que estos 
sistemas y plataformas favorece una retroalimentación inmediata con el profesor, mientras que el 84\% piensa que además fomenta el trabajo en grupo. Como valoración global del recurso educativo, se obtuvo en las encuestas que el 86.5\% está muy de acuerdo o de acuerdo con esta tecnología, obteniendo una valoración media de 4.33.

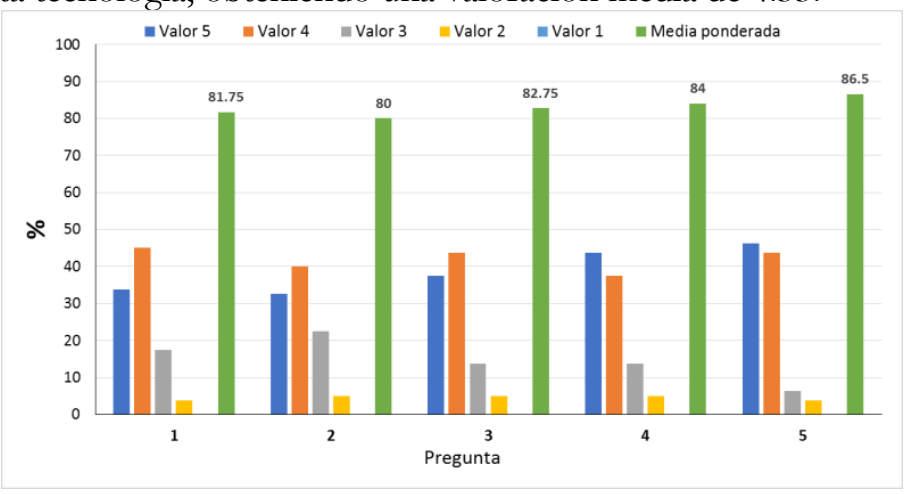

4. Resultados obtenidos de la encuesta de opinión anónima.

\section{UTILIDAD/ANÁLISIS}

Como se ha expuesto anteriormente, con esta experiencia se ha comprobado la eficacia pedagógica que tiene la metodología planteada frente al sistema tradicional de clases magistrales.

Mediante el uso de ordenadores, tablets, smartphones o clickers como sistemas de respuesta interactiva para realizar cuestionarios u otras actividades evaluativas, se ha logrado un mejor aprovechamiento del tiempo limitado que el alumno tiene en las prácticas de laboratorio. Esta metodología propicia que los alumnos acudan a la práctica más preparados, ya que saben que al inicio de dicha práctica se les realizará un cuestionario sobre los contenidos del guion. Esto fomenta una actitud más activa del alumno y permite el desarrollo adecuado de la práctica en el tiempo establecido para la sesión.

Por otro lado, el alumno adopta una actitud más activa, el profesor dispone de retroalimentación inmediata, lo que le permite centrar la explicación en conceptos mal entendidos por los alumnos y, además, la metodología proporciona una herramienta de evaluación para el profesor, y de autoevaluación para el alumno. Un aspecto muy importante del uso de esta tecnología, es la ventaja de darle una retroalimentación inmediata al alumno sobre su respuesta, es decir, el alumno conoce en tiempo real cuál ha sido su fallo y por qué lo ha cometido.

\section{CONCLUSIONES/DISCUSIÓN}

En este estudio se ha implementado una nueva metodología de trabajo en laboratorio, fomentando el aprendizaje proactivo del alumno. La disponibilidad de laboratorios es muy limitada, por haber muchos grupos y muchas titulaciones con la asignatura de Fundamentos Físicos de la Ingeniería en el primer curso. El objetivo de que las sesiones lectivas sean más interactivas y el estudio previo fuera del aula más efectivo se ha cumplido. Las diferentes acciones llevadas a cabo han mejorado el paradigma enseñanza-aprendizaje, fomentando la utilización de recursos educativos destinados a la innovación dentro y fuera del aula.

En conclusión, esta metodología fomenta que el alumno acuda a las sesiones de prácticas de laboratorio con el guion estudiado y una actitud activa. Por otro lado, esta metodología permite darle una retroalimentación inmediata al alumno sobre su respuesta, es decir, el alumno conoce en tiempo real cual ha sido su fallo y porqué lo ha cometido, observándose una mayor motivación e involucración en el proceso de aprendizaje.

\section{AGRADECIMIENTOS.}

Este trabajo presenta los resultados obtenidos en un Proyecto de Innovación de Grupos Docentes, convocatoria 16-17, financiado por Vicerrectorado de Estudios Postgrado y Formación Continua de la Universidad de Córdoba. 
BIBLIOGRAFÍA

BRIZ-PONCE, L., PEREIRA, A., CARVALHO, L., JUANES-MÉNDEZ, J. A., GARCÍA-PEÑALVO, F. J. “Learning with mobile technologies-students' behavior", Computers in Human Behavior, 2017/72, p. 612-620.

KATZ, L., HALLAM, M. C., DUVALL, M. M., \& POLSKY, Z. "Considerations for using personal wi-fi enabled devices as "clickers" in a large university class", Active Learning in Higher Education, 2017/18(1), p. 25-35.

KULIKOVSKIKH, I. M., PROKHOROV, S. A., \& SUCHKOVA, S. A. "Promoting collaborative learning through regulation of guessing in clickers", Computers in Human Behavior, 2017/75, 81-91.

LUCKE, T., DUNN, P. K., CHRISTIE, M. “Activating learning in engineering education using ICT and the concept of Flipping the classroom”, European Journal of Engineering Education, 2017/42(1), p. 45-57.

SHAPIRO, A. M., SIMS-KNIGHT, J., O'RIELLY, G. V., CAPALDO, P., PEDLOW, T., GORDON, L., MONTEIRO, K. "Clickers can promote fact retention but impede conceptual understanding: The effect of the interaction between clicker use and pedagogy on learning", Computers and Education, 2017/111, p. 44-59.

WAHEED, M., KAUR, K., KUMAR, S. “What role does knowledge quality play in online students' satisfaction, learning and loyalty? an empirical investigation in an eLearning context”, Journal of Computer Assisted Learning, 2016/32(6), p. 561-575. 